\title{
Caracterización clínico y epidemiológica en pacientes con infección por Enterobacteriaceae productoras de B lactamasas de espectro extendido (BLEE), Hospital Escuela Universitario, Tegucigalpa, Honduras, Año 2013
}

Lelany Pineda-Garcia ${ }^{1}$

Edgardo Tzoc ${ }^{2}$

Maria Felix Rivera ${ }^{3}$

Linda Herrera ${ }^{4}$

Marco Moncada ${ }^{5}$

\section{RESUMEN}

Antecedentes. La prevalencia de Enterobacteriaceae productoras de B lactamasas de espectro extendido (BLEE) se ha incrementado a nivel mundial aumentado el riesgo de mortalidad en los pacientes infectados con este tipo de bacterias. Objetivo: Determinar las características clínico y epidemiológica en pacientes con infección por Enterobacteriaceae productoras de B lactamasas de espectro extendido (BLEE) en el Hospital Escuela Universitario. Tegucigalpa, Honduras. Año 2013.Metodología: Estudio transversal descriptivo en pacientes con cultivos positivos por Escherichia coli y Klebsiella pneumoniae productoras de BLEE. Los cultivos positivos se identificaron a través de los registros del laboratorio de bacteriología del hospital de junio a agosto del 2013. Las muestras positivas fueron analizadas por medio del método Kirby-Bauer y la prueba comercial E-test para Concentración Mínima Inhibitoria (MIC). Se revisaron los expedientes clínicos para obtener la información clínico y epidemiológica. El análisis de la información se realizó en Epi info 7. Resultados: en

\footnotetext{
${ }^{1}$ Beneficiaria de una beca sustantiva de la DICYP. Coordinadora del proyecto del Instituto de Investigación de Ciencias de Salud, Facultad de Ciencias Médicas e investigadora del Laboratorio de Investigación de Enfermedades Tropicales, Escuela de Microbiología. Facultad de Ciencias. UNAH: lelanypineda@unah.edu.hn

2 Beneficiario de una beca sustantiva de la DICYP. Profesor de la sección de Bacteriología., Escuela de Microbiología, Facultad de Ciencias. UNAH: etzoc2000@yahoo.com

${ }^{3}$ Beneficiaria de una beca sustantiva de la DICYP. Profesora del Departamento de Fisiología y Coordinadora de Investigación y Vinculación, Maestría en Epidemiología. Facultad de Ciencias Médicas. UNAH: mariafelixrivera@yahoo.es

${ }^{4}$ Beneficiaria de una beca sustantiva de la DICYP. Laboratorio de Bacteriología. Hospital Escuela Universitario.

${ }^{5}$ Beneficiario de una beca sustantiva de la DICYP. Laboratorio de Bacteriología. Hospital Escuela Universitario
} 
el periodo se recolectaron 50 muestras de cultivos positivos por E. coli y 49 muestras por $\mathrm{K}$ pneumoniae. La media de edad de los pacientes con $\mathrm{E}$. coli fue 33.6 años \pm 26.5 1DE y 26.5 años $\pm 26.4 \mathrm{DE}$ con $\mathrm{K}$. pneumoniae. Las muestras fueron recolectada a los 10.0 días en promedio de hospitalización para los pacientes con E. coli y 21.8 días para los de K. pneumoniae y las diferencias fueron significativas $(p=0.02)$. E. coli presentó resistencia a 9 de los 11 antibióticos, K. pneumoniae a 10 de los 11 antibióticos estudiados y las diferencias fueron significativas ( $p=0.00002)$, el tipo de muestra más frecuente donde se aisló $\mathrm{E}$. coli fue de orina $(62.0 \%)$ y K. pneumoniae solo un $34.7 \%$ de orina seguido de hemocultivo (16.3\%) y UCI fue donde se aisló el mayor porcentaje de K. pneumoniae en cambio E. coli fue en consulta externa. Conclusiones/Recomendaciones: los resultados de este estudio muestran la existencia de Enterobacteriaceae BLEE, altamente resistentes, esencialmente a las cefalosporinas de tercera generación y se deben redefinir los esquemas de antibioticoterapia para el mejoramiento de la calidad de atención médica hospitalaria.

Palabras claves: factores epidemiológicos, betalactamasas, infección. E. coli, K. pneumoniae

\section{ABSTRACT}

Previous studies demonstrate that the prevalence of Enterobacteriaceae producing $B$ spread spectrum lactamases (ESBL) has increased worldwide increasing the risk of mortality in patients infected with this type of bacteria. Objective: To determine the clinical and epidemiological characteristics in patients with Enterobacteriaceae infection producing B spread-spectrum lactamases (ESBL) at the University's School Hospital in Tegucigalpa, Honduras in the year 2013. Methodology: Descriptive cross-sectional study in patients with ESBL-producing Escherichia coli and Klebsiella pneumoniae positive cultures. Positive cultures were identified through hospital bacteriology laboratory records from June to August 2013. Positive samples were analyzed using the Kirby-Bauer method and the commercial E-test for Minimum Inhibitory Concentration (MIC). Clinical records were reviewed to obtain clinical and epidemiological information. Data analysis was performed in Epi info 7. Results: 50 samples of E. coli positive cultures and 49 samples of $\mathrm{K}$ pneumoniae were collected during the period. The mean age of patients with $E$. coli was 33.6 years $\pm 26.51 \mathrm{DE}$ and 26.5 years $\pm 26.4 \mathrm{DE}$ with $\mathrm{K}$. pneumoniae. Samples were collected at 10.0 days in average hospitalization for patients with $\mathrm{E}$. coli and 21.8 days for $\mathrm{K}$. pneumoniae patients and differences were significant $(p=0.02)$. E. coli was resistant to 9 of the 11 antibiotics, 
K. pneumoniae to 10 of the 11 antibiotics studied and the differences were significant $(p=0.00002)$, the most frequent type of sample where $E$. coli was isolated was urine $(62.0 \%)$ And K. pneumoniae only $34.7 \%$ of urine followed by hemoculture $(16.3 \%)$ and ICU was where the highest percentage of $\mathrm{K}$. pneumoniae was isolated whereas E. coli was in outpatient clinic. Conclusions / Recommendations: The results of this study show the existence of BLEE Enterobacteriaceae, highly resistant, essentially to the third generation cephalosporins and should be redefined the antibiotic therapy schemes for the improvement of the quality of hospital medical care.

Key words: epidemiological factors, betalactamasas, infection. E. coli, K. pneumoniae 


\section{INTRODUCCIÓN}

La Familia Enterobacteriaceae comprende un grupo de bacilos Gram negativos, distribuidos en la naturaleza y que también son parte de la flora normal del ser humano (intestinos, piel, tracto respiratorio). La importancia clínica de las Enterobacteriaceae está asociada con enfermedades específicas causadas por géneros y especies bien identificadas, diferenciándose en infecciones de tipo intestinal y extra intestinal. (Murray PR, 2007)

El valor en el campo de la medicina de las especies oportunistas es principalmente que están asociadas a la atención en los servicios de salud, destacándose algunos géneros y cepas de Enterobacteriaceae por su habilidad de persistir en ciertos ambientes hospitalarios. (Araya FC, 2006) (Escalante-Montoya JC, 2013)

Las infecciones asociadas a la atención en salud (IASS) se consideran en la actualidad como un verdadero problema de salud pública afectando a más del $15 \%$ de los pacientes hospitalizados. (DeLisle S, 2003)

En los Estados Unidos se calcula que se producen alrededor de dos millones de IAAS cada año y 77,000 muertes, con un costo asociado de 15,000 USD por paciente afectado, con un costo acumulado de entre 5 a 10 mil millones de USD. (Araya FC, 2006)

Dentro de estas cepas destacan en la actualidad las que poseen la capacidad de producir beta lactamasas de espectro extendido BLEE, siendo Escherichia coli y Klebsiella pneumoniae las principales.(Rupp M, 2003)

Las Enterobacteriaceae productoras de BLEE presentan desafíos únicos en el sistema de salud, ya que estas cepas son capaces de inactivar penicilinas, cefalosporinas de amplio espectro y monobactams. Estas BLEE se derivan de enzimas tipo TEM, SHV y CTX-M, codificadas generalmente en plásmidos por lo cual son altamente transferibles entre diferentes especies e inclusive géneros. Aunque no se conoce su verdadera incidencia, se sabe que está en aumento y entre un 10 a un $40 \%$ de las cepas de Escherichia coli y K. pneumoniae son productoras de BLEE. (Zemelman R, 2002)

Los primeros casos de cepas resistentes a estas cefalosporinas de tercera generación se documentó primero en Europa en Alemania en 1983, desde entonces la apari- 
ción de cepas específicas que expresan determinados genes de $\beta$ lactamasas ha ido en aumento, siendo en la actualidad identificadas más de 350 diferentes beta lactamasas y algunas 150 con distribución mundial. (Zemelman R, 2002) ( Giamarellou H, 2005)

Asimismo en Estados Unidos la prevalencia es en un rango amplio de 0 a $25 \%$, con un promedio nacional del $3 \%$.(Zemelman R, 2002)

En Honduras, un estudio en el Instituto Hondureño de Seguridad Social (IHSS) del 2006 al 2009, se registraron 4,812 aislamiento procedente de urocultivos, hemocultivos y de secreciones varias y diversas fuentes encontrando que las bacterias gram negativas aisladas fueron las más frecuentes y de ellas la más común fue la Klebsiella pneumoniae, Escherichia coli y presentando una alta resistencia a quinolonas. (Padgett D, 2011). El país no cuenta con muchos estudios en esta línea y mucho menos estudios de Enterobacterias multirresistentes por lo que esta investigación tuvo como objetivo determinar las características clínico y epidemiológica en pacientes con infección por Enterobacteriaceae productoras de ßlactamasas de espectro extendido (BLEE) en el Hospital Escuela Universitario. Tegucigalpa, Honduras. Año 2013.

\section{METODOLOGÍA}

Se diseñó un estudio de tipo descriptivo, transversal. Se recolectaron 50 cepas de Escherichia coli y 49 cepas de Klebsiella pneumoniae ya identificadas como BLEE aisladas de pacientes del Hospital Escuela Universitario de junio a agosto del 2013. No se hizo un muestreo sino que se tomó el universo, todas las bacterias E. coli y K. pneumoniae aislada en el periodo con el fenotipo de BLEE. luego fueron trasladas al Laboratorio de Enfermedades Tropicales de la Escuela de Microbiología UNAH, se les realizó aislamiento, identificación y susceptibilidad a antibióticos (Amoxicilina +Ac. Clavulanico, Cloranfenicol, Gentamicina, Norfloxacina Tetraciclina, Trimetropin sulfametoxazol, Cefoxitin, Cefpodoxime, Ceftazidime, Cefotaxima, Ceftriaxona).Se utilizó el método Kirby-Bauer y la prueba comercial E-test para Concentración Mínima Inhibitoria (MIC). Se diseñó una ficha clínica donde se registraron las siguientes variables como ser: edad, genero, procedencia, diagnóstico clínico, comorbilidad, sala de atención, días de hospitalización, lugar de aislamiento de la bacteria y tipo de muestra. La información clínica-epidemiológica se obtuvo de los expedientes clínicos de hospitalización. Para el procesamiento de la información se utilizó Epi info 7.2 y 
Excel donde su obtuvieron frecuencias, porcentajes, medidas de tendencia central, para comparaciones entre las dos cepas t de student y chi cuadrado cuando se requirió, y se consideró un nivel de significancia de $p<$ de 0.05 .

\section{RESULTADOS}

\section{Caracterización sociodemográfica}

Los pacientes en los cuales se les aisló Escherichia coli con fenotipo BLEE tenían una edad promedio de 33.6 años $\pm 25.81 \mathrm{DE}$ y en los pacientes donde se aisló Klebsiella pneumoniae con fenotipo BLEE fue de 26.9 años \pm 26.7 1DE y las diferencias encontradas no fueron estadísticamente significativas.

Gráfico 1. Etapa de la vida según el tipo de Enterobacteria Aislada fenotipo BLEE de pacientes del Hospital Escuela Universitario. Junio a Agosto 2013.

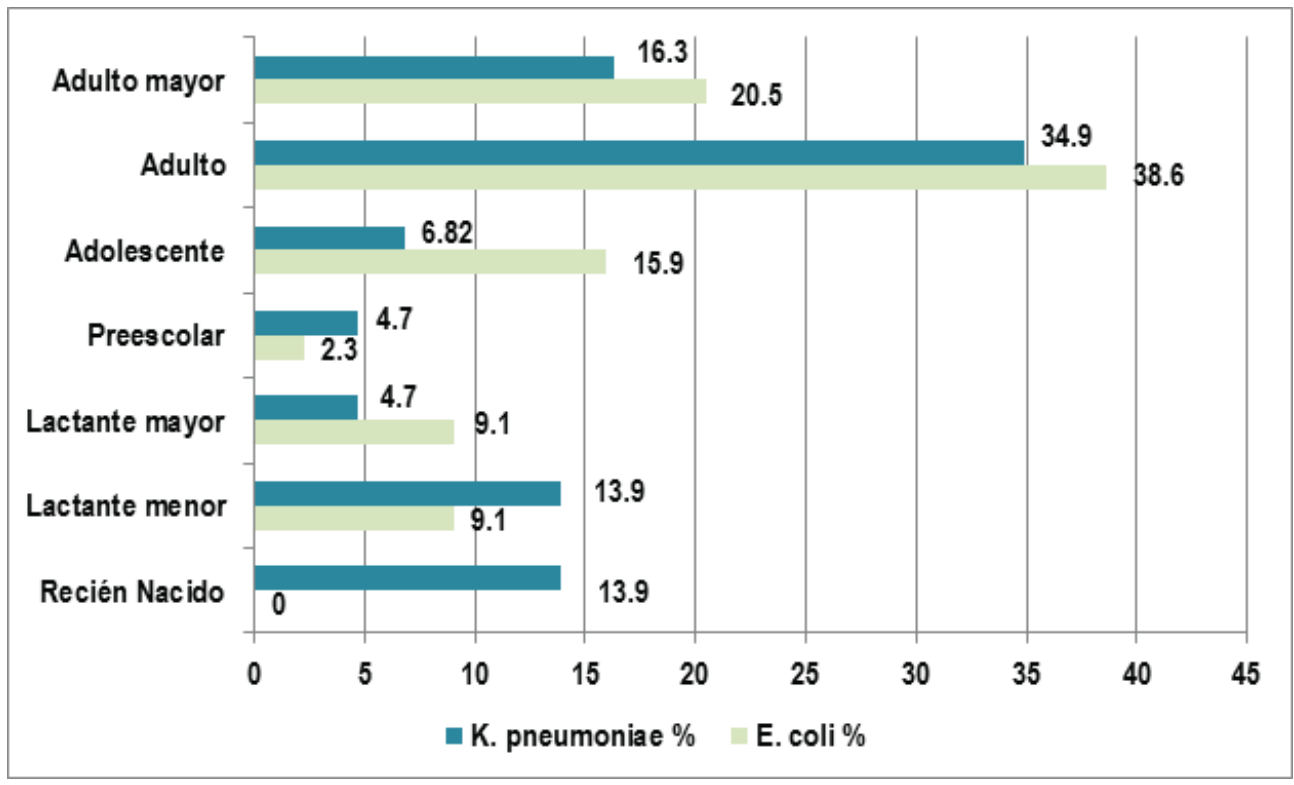

Las etapas de la vida donde se aisló E. coli fue en más del $50 \%$ en el adulto y el adulto mayor, así mismo K. pneumoniae con la diferencia de que esta última se aisló también en recién nacidos. (Ver gráfico 1) 
Gráfico 2. Distribución del tipo de enterobacteria aislada fenotipo BLEE según género en pacientes del Hospital Escuela Universitario. Junio a Agosto 2013.

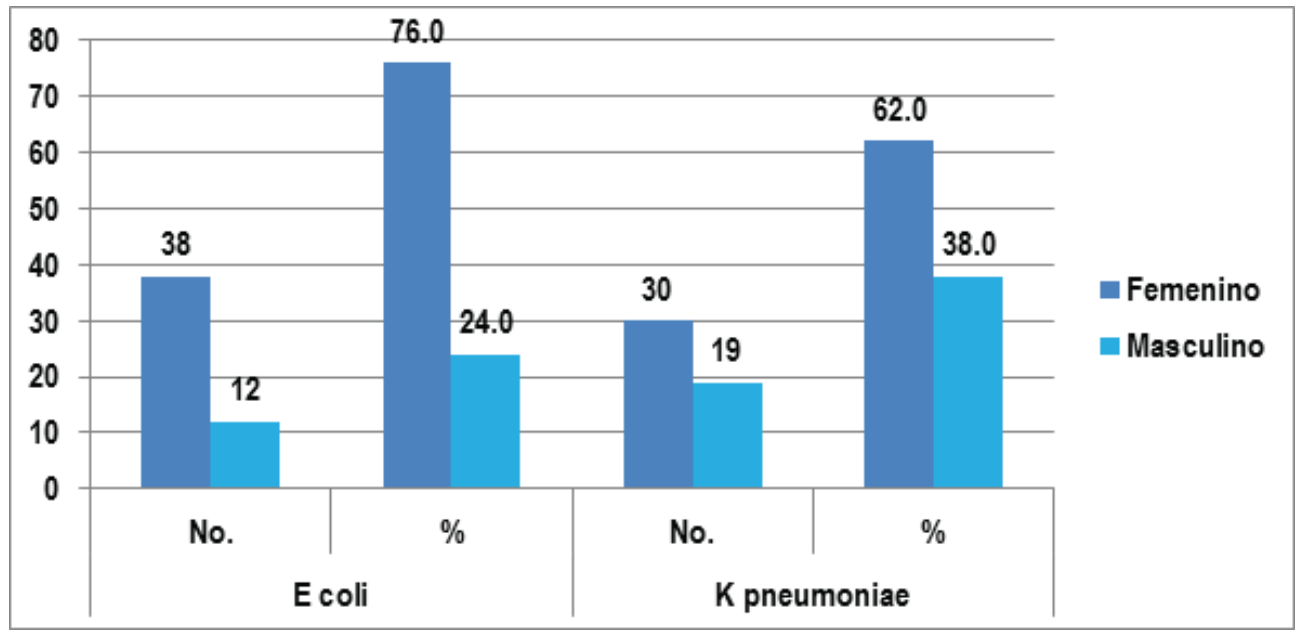

En cuanto al género, el aislamiento de $\mathrm{E}$. coli fue más frecuente en el femenino $(76.0 \%)$ a diferencia de $\mathrm{K}$. pneumoniae que fue en un porcentaje menor $61.2 \%$ aunque las diferencias no fueron estadísticamente significativas $(p=0.17)$, como se observa en el gráfico 2.

Se logró obtener información de los expedientes clínicos, sin embargo se tuvo perdida de la información ya que en algunos expedientes no estaba consignada.

En cuanto a la procedencia, más de la mitad de los pacientes en los cuales se les aisló E. coli procedían Francisco Morazán (67.9\%), a diferencia de los pacientes con K. pneumoniae que tuvo una mayor distribución como se puede observar en el cuadro 1.

El 37\% de los pacientes donde se le aisló E. coli se tomó la muestra en el momento de su ingreso y ninguna de las cepas de K. pneumoniae estudiadas se aisló al ingreso de pacientes sino después de las 48 horas de estadía hospitalaria. Para E. coli el tiempo de recolección de muestra en sala fue en una media de 10.0 días $\pm 16.11 \mathrm{DE}$ y para K. pneumoniae fue mayor de 21.8 dias $\pm 18.91 \mathrm{DE}$ y la diferencias de medias fue estadísticamente significativa $(p=0.02)$ 
Cuadro 1. Distribución de procedencia por departamento según el tipo de enterobacteria aislada fenotipo BLEE de pacientes del Hospital Escuela Universitario. Junio a Agosto 2013.

\begin{tabular}{|l|c|c|}
\hline \multirow{2}{*}{} & \multicolumn{2}{|c|}{ Tipo de bacteria } \\
\cline { 2 - 3 } & $\begin{array}{c}\text { E. coli } \\
\mathrm{n}=37\end{array}$ & $\begin{array}{c}\text { K. pneumoniae } \\
\mathrm{n}=27\end{array}$ \\
\cline { 2 - 3 } & No. $(\%)$ & No. $(\%)$ \\
\hline Departamento & & \\
\hline Choluteca & $10(3.6)$ & $3(11.1)$ \\
\hline Comayagua & $4(14.3)$ & $4(14.8)$ \\
\hline El Paraíso & $1(3.6)$ & $2(7.4)$ \\
\hline Francisco Morazán & $19(67.9)$ & $10(37.0)$ \\
\hline Islas de la Bahía & $0(0.0)$ & $1(3.7)$ \\
\hline La Paz & $3(10.7)$ & $0(0.0)$ \\
\hline Olancho & $0(0.0)$ & $6(22.2)$ \\
\hline Valle & $0(0.0)$ & $1(3.7)$ \\
\hline
\end{tabular}

Cuadro 2. Cultivo según el tipo de Enterobacteria BLEE. Hospital Escuela Universitario. Junio a Agosto 2013.

\begin{tabular}{|l|c|c|}
\hline \multirow{2}{*}{} & \multicolumn{2}{|c|}{ Tipo de bacteria } \\
\cline { 2 - 3 } & $\begin{array}{c}\text { E. coli } \\
\mathrm{N}=50\end{array}$ & $\begin{array}{c}\text { K. pneumoniae } \\
\mathrm{n}=49\end{array}$ \\
\cline { 2 - 3 } & No.( \%) & No.( $\%)$ \\
\hline Cultivo & & \\
\hline Orina & $31(62.0)$ & $17(34.7)$ \\
\hline Secreción de herida quirúrgica & $6(12.0)$ & $3(6.1)$ \\
\hline Hemocultivo & $4(8.0)$ & $8(16.3)$ \\
\hline Secreción absceso & $4(2.0)$ & $0(2.4)$ \\
\hline Catéter & $1(2.0)$ & $5(10.2)$ \\
\hline Coprocultivo & $1(2.0)$ & $0(0.0)$ \\
\hline Líquido pleural & $1(2.0)$ & $0(0.0)$ \\
\hline Secreción hueso & $1(2.0)$ & $4(8.2)$ \\
\hline Jugo gástrico & $0(0.0)$ & $2(4.1)$ \\
\hline LCR & $0(0.0)$ & $1(2.0)$ \\
\hline Liquido peritoneal & $0(0.0)$ & $1(2.0)$ \\
\hline Secreción quemadura & $0(0.0)$ & $1(2.0)$ \\
\hline Secreción de ulcera & $0(0.0)$ & $2(4.1)$ \\
\hline Secreción vaginal & $0(0.0)$ & $1(2.0)$ \\
\hline Secreción de Traqueotomía & $0(0.0)$ & $3(6.1)$ \\
\hline
\end{tabular}


De orina se aisló la mayor cantidad de E. coli (62.0\%) y de K. pneumoniae en un $36.4 \%$. De los hemocultivos el mayor porcentaje fue K. pneumoniae en un $21.2 \%$ y de Escherichia coli en un $8.0 \%$. (Ver cuadro 2)

Los pacientes con aislamiento de E. coli tuvieron una media en días de hospitalización $10.0 \pm 16.17$ días y para los de Klebsiella pneumoniae fue de $21.86 \pm 18.9$ días $y$ las diferencias fueron estadísticamente significativas $(p=0.002)$.

Cerca del $40 \%$ de todos los pacientes habían tenido hospitalizaciones previas.

Cuadro 3.Diagnóstico clínico según el tipo de Enterobacteria BLEE. Hospital Escuela Universitario. Junio a Agosto 2013.

\begin{tabular}{|l|c|c|}
\hline & $\begin{array}{c}\text { E. coli } \\
\mathrm{n}=30\end{array}$ & $\begin{array}{c}\text { K. pneumoniae } \\
\mathrm{n}=36\end{array}$ \\
\cline { 2 - 3 } & No. (\%) & No. (\%) \\
\hline Diagnóstico & & \\
\hline Infección del tracto urinario & $18(60.0)$ & $12(33.3)$ \\
\hline Herida quirúrgica infectada & $4(13.3)$ & $4(11.1)$ \\
\hline Absceso & $2(6.6)$ & $0(0.0)$ \\
\hline Neumonía & $2(6.7)$ & $3(8.3)$ \\
\hline Fistula enterocutánea & $1(3.3)$ & $0(0.0)$ \\
\hline Fractura infectada & $1(3.3)$ & $6(16.6)$ \\
\hline Gastroenteritis & $1(3.3)$ & $0(0.0)$ \\
\hline Sepsis & $1(3.3)$ & $2(5.6)$ \\
\hline Hidrocefalia & $0(0.0)$ & $1(2.8)$ \\
\hline Inmunosupresión & $0(0.0)$ & $5(13.9)$ \\
\hline Pie diabético & $0(0.0)$ & $1(2.8)$ \\
\hline Quemadura & $0(0.0)$ & $2(5.6)$ \\
\hline
\end{tabular}

Los diagnósticos de los pacientes donde se aisló E. coli estaba asociado más a enfermedades de vías urinarias incluyendo urosepsis y K. pneumoniae a sepsis, heridas quirúrgicas infectadas y neumonía por uso de ventilador mecánico. (Ver cuadro 3) 


\section{Cuadro 4. Comorbilidad según el tipo de Enterobacteria BLEE. Hospital Escuela Universitario. Junio a Agosto 2013}

\begin{tabular}{|l|c|c|}
\hline \multirow{2}{*}{} & \multicolumn{2}{|c|}{ Tipo de bacteria } \\
\cline { 2 - 3 } & $\begin{array}{c}\text { E. coli } \\
\mathrm{n}=21\end{array}$ & $\begin{array}{c}\text { K. pneumoniae } \\
\mathrm{n}=16\end{array}$ \\
\hline & No. $(\%)$ & No. $(\%)$ \\
\hline Comorbilidad & & \\
\hline Insuficiencia renal & $8(38.1)$ & $3(18.8)$ \\
\hline Diabetes Mellitus tipo 2 & $5(23.8)$ & $3(18.8)$ \\
\hline Enfermedad cerebro vascular & $5(23.8)$ & $5(31.3)$ \\
\hline Demencia & $1(4.8)$ & $0(0.0)$ \\
\hline Neoplasia & $1(4.8)$ & $4(25.0)$ \\
\hline Desnutrición & $1(4.8)$ & $1(6.3)$ \\
\hline
\end{tabular}

En relación a la comorbilidad mas frecuentes encontradas, $38.1 \%$ insuficiencia renal y $23.8 \%$ Diabetes Mellitus tipo 2 en los pacientes donde se les aisló E. coli BLEE, $31.3 \%$ enfermedad Cerebro Vascular y $18.8 \%$ Diabetes Mellitus en igual porcentaje insuficiencia renal en los pacientes donde se aisló K. pneumoniae BLEE (Ver cuadro 4)

En relación a la ubicación del paciente al momento de la toma de muestra, se observó que el $24 \%$ delas muestras donde se aísló E. coli BLEE fueron tomadas en consulta externa y KI. pneumoniae en un porcentaje menor de un $8.0 \%, 14.0 \%$, en la sala de emergencia las dos bacteria, el resto de las bacterias se aislaron ya estando el paciente en salas, el mayor porcentaje en salas de $\mathrm{E}$. coli se asilaron en cirugía $14.0 \%$, Medicina Interna 14.0\%, Terapia Renal en un 10.0\%, entre consulta externa, emergencia y estas tres salas suman más del $75.0 \%$ de aislamiento. el mayor porcentaje de K. pneumoniae BLEE en los pacientes estudiados fue aislado en salas; el $20.4 \%$ fue asilado de UCI, $14.0 \%$ de emergencia, 14.0 de Medicina Interna, 10.0\% de pediatría y $10.0 \%$ de ortopedia, que constituyen el $68.4 \%$ de los aislamiento por $\mathrm{K}$ pneumoniae. Como se puede observar en el gráfico 3. 
Gráfico 3. Consulta Externa y tipo de sala según el tipo de Enterobacteria BLEE de pacientes del Hospital Escuela Universitario. Junio a Agosto 2013.

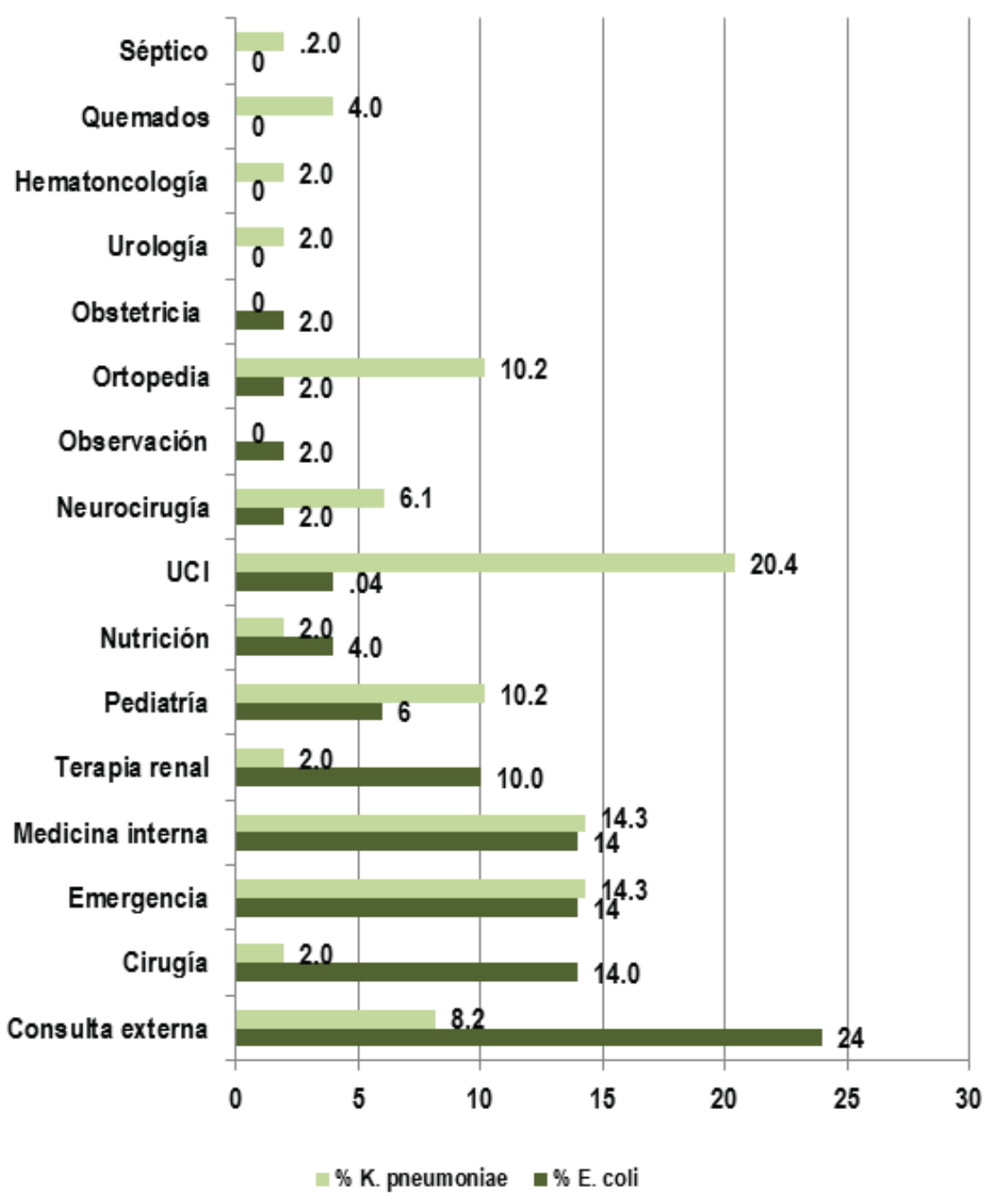

La resistencia antibiótica tanto de E. coli como K. pneumoniae fue en un $100 \%$ para Cefotaxima y Ceftriaxona, con resistencia muy altas de más del $90 \%$ también para Amoxicilina + ácido Clavulánico, Cefoxitin, Cefpodoxime, Ceftazidime de ambas bacterias estudiadas y entre $70 \%$ a $90 \%$ resistencia de la E. coli para Norfloxacina, Trimetropin Sulfametoxazol, Tetraciclina y Gentamicina, no así es el comportamiento de la K. pneumoniae donde el $96 \%$ fue resistente al Trimetropin Sulfametoxazol y en 
igual porcentaje para Tetraciclina y en menor porcentaje ligeramente menor a la Gentamicina en un $94 \%$ a diferencia del Cloranfenicol donde solo el $6.0 \%$ de los aislamientos de E. coli fueron resistentes y el $54.0 \%$ de K. pneumoniae. (Ver cuadro 5)

Cuadro 5. Resistencia antibiótica según el tipo de enterobacteria BLEE. Hospital Escuela Universitario. Junio a Agosto 2013

\begin{tabular}{|l|c|c|}
\hline \multirow{2}{*}{} & \multicolumn{2}{|c|}{ Tipo de bacteria } \\
\cline { 2 - 3 } & E. coli & K. pneumoniae \\
\cline { 2 - 3 } & No. $(\%)$ & No. $(\%)$ \\
\hline Antibiótico & & \\
\hline Cefotaxima & $50(100.0)$ & $49(100.0)$ \\
\hline Ceftriaxona & $50(100.0)$ & $49(100.0)$ \\
\hline Amoxicilina +Ac. Clavulánico & $49(98.0)$ & $45(90.0)$ \\
\hline Cefoxitin & $48(96.0)$ & $50(100.0)$ \\
\hline Cefpodoxime & $48(96.0)$ & $49(98.0)$ \\
\hline Ceftazidime & $48(96.0)$ & $49(98.0)$ \\
\hline Norfloxacina & $42(84.0)$ & $35(70.0)$ \\
\hline Trimetropin Sulfametoxazol & $41(82.0)$ & $48(96.0)$ \\
\hline Tetraciclina & $38(76.0)$ & $48(96.0)$ \\
\hline Gentamicina & $36(72.0)$ & $47(94.0)$ \\
\hline Cloranfenicol & $3(6.0)$ & $27(54.0)$ \\
\hline & & \\
\hline
\end{tabular}

Durante la hospitalización los pacientes con E. coli recibieron en promedio de 2.0 antibióticos y un mínimo de uno y máximo de 6 y para K. pneumoniae fue un mínimo de uno y un máximo de cuatro. Y las diferencias no fueron estadísticamente significativas.

Los pacientes con aislamiento de E. coli BLEE el $73.1 \%$ recibió Betalactámicos y en K. pneumoniae BLEE un porcentaje menor de un $66.7 \%$, las diferencias no fueron estadísticamente significativas $(p=0.89)$ y en menor porcentaje otros antibióticos como se observan en el cuadro 6.

Los pacientes estudiados habían sido remitidos de otros hospitales casi en el mismo porcentaje aproximadamente un $30 \%$ de los que se les aisló Escherichia coli como de los que se aisló Klebsiella pneumoniae 
Cuadro 6. Grupo y antibiótico recibido según el tipo de Enterobacteria BLEE. Hospital Escuela Universitario. Junio a Agosto 2013

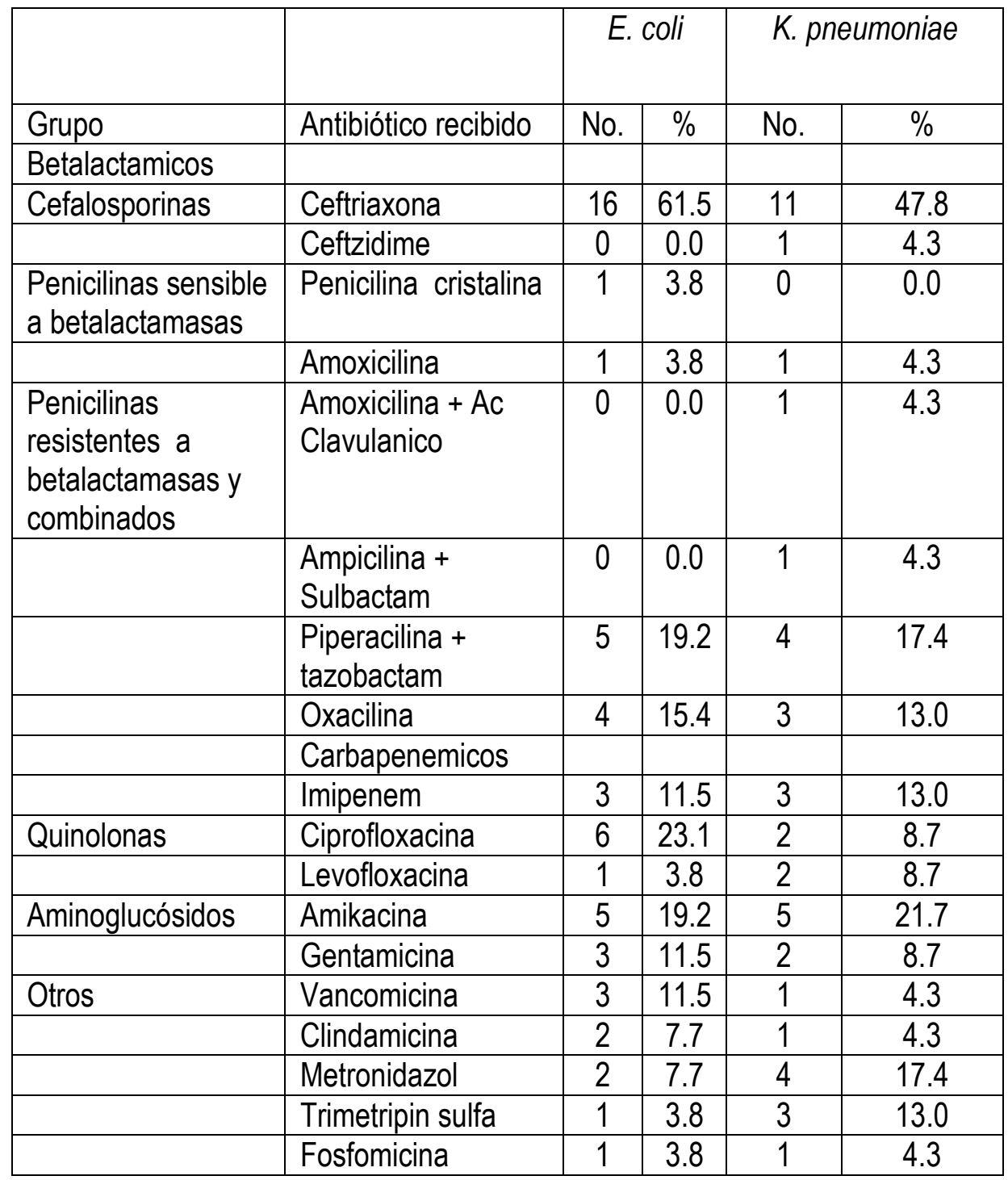

* Cada fila es independiente por lo que no suma a $100 \%$.

El número de antibióticos recibidos en la sala para pacientes que se les aisló E. coli fue de $2.1 \pm 1.3$ y para K. pneumoniae fue de $2.0 \pm 0.8$ las diferencias no fueron estadísticamente significativos $(p=0.9)$. 


\section{DISCUSIÓN}

La producción de BLEE por diversas bacterias patógenas es de mucha importancia clínica ya que constituye un trascendental problema en pacientes hospitalizados debido a las implicaciones clínicas, terapéuticas y el incremento en los costos de tratamiento. Así observamos en nuestro estudio que durante la hospitalización los pacientes con Escherichia coli BLEE recibieron en promedio de 2.0 antibióticos y un mínimo de uno y máximo de 6 y para Klebsiella pneumoniae fue un mínimo de uno y un máximo de cuatro. Sin embargo las diferencias no fueron estadísticamente significativas. (Schwaber MJ, 2006) (Seaman I,2011)

En este estudio se recolectaron 50 cepas de E. coli BLEE y 49 de K. pneumoniae BLEE en solamente tres mes en el Hospital estudiado lo que denota la presencia de la mismas y la oportunidad para futuras investigaciones asi mismo como intervenciones.

A un tercio de los pacientes donde se le aisló Escherichia coli la muestra se tomó al momento de su ingreso y de donde se aisló Klebsiella pneumoniae se tomaron las muestras después de las 48 horas de estadía hospitalaria. Estudios en Africa hacen mención de la obtención de cepas BLEE en la comunidad para E. coli. (Alabi AS, 2013)

La presencia de enterobacterias BLEE adquirida en la comunidad se ha descrito en varios estudios países del mundo tanto en los desarrollados como ser Estados Unidos (Alanis AJ,2005) (Muñoz Bellido JL, 2004) y también en países en vías de desarrollo en estos últimos se asocia a la toma decisiones terapéuticas en forma empírica así como a la escasa disponibilidad de antibióticos alternativos de segunda línea, también al complejo escenario de enfermedades infecciosas que se enfrenta en nuestros países. (Perez C, 2003) (Alabi AS, 2013)

Así observamos en este estudio que el antibiótico más utilizado en el tratamiento de los pacientes estudiados fue ceftriaxona del cual ambas bacterias no tenían ninguna suceptibilidad lo que constituye un aumento en los costos hospitalarios como también se encontró una baja suceptibilidad a fluorquinolonas iguales hallazgos fueron demostrados en un estudio realizado en un hospital rural al sur de Mozambique .(Mandomando I, 2010)

Es de hacer notar la relación del aislamiento de Escherichia coli BLEE en urocultivos 
y en pacientes del género femenino a diferencia de Klebsiella pneumoniae que se aisló en un mayor porcentaje de hemocultivo aunque las diferencias no fueron estadísticamente significativas esto se debe a la asociación de la E. coli con infecciones urinarias y estas son más frecuente en el sexo femenino sin embargo a los 65 años la relación se invierte debido a la retención e incontinencia urinaria por alteraciones en la próstata.(Dielubanza EJ, 2011). Por lo tanto los diagnósticos de los pacientes donde se aisló Escherichia coli fueron enfermedades de vías urinarias incluyendo urosepsis y Klebsiella pneumoniae a sepsis, heridas quirúrgicas infectadas y neumonía por uso de ventilador mecánico.

La comorbilidad de los pacientes donde se les aisló Escherichia coli en un tercio fue insuficiencia renal y dos de cada diez Diabetes Mellitus y para Klebsiella pneumoniae fue diferente en un tercio tenían enfermedad Cerebro Vascular y ligeramente mayor el porcentaje con Diabetes Mellitus. Un estudio realizado en Salamanca España encontró, en pacientes críticos fue más frecuente la $\mathrm{K}$. pneumoniae y en foco urinario el más frecuente fue E. coli (Perianes-Díaza ME,2014)

También en España se encontró que el 39.8\% presentaban enfermedades asociadas con diabetes mellitus, cardiopatía o enfermedad pulmonar obstructiva crónica. (Martínez M, 2013)

Otro estudio en Colombia, en infecciones del tracto urinario, se encontró con gran preocupación la resistencia creciente de los microorganismos causantes frente a los antibióticos de primera línea y la aparición de cepas resistentes productoras de betalactamasas de espectro extendido en la comunidad y se encontró comorbilidades mas frecuentes como hipertensión arterial en un primer lugar $(25.2 \%)$ y diabetes mellitus (20.9\%) en segundo lugar.(Leal AL, 2013).

En nuestra investigación se aisló con mayor frecuencia en Recién Nacidos y en pacientes de $\mathrm{UCl}$ solo $\mathrm{K}$ pneumoniae BLEE estos resultados están apoyados por Oliveira. Hace mención de que la población pediátrica representa uno de los grupos más vulnerables, siendo los recién nacidos los más afectados, especialmente aquellos hospitalizados en las salas de cuidado intensivo neonatal. Esto debido al uso en gran medida de aparatos e instrumentos médicos, antimicrobianos y también por la falta de maduración del sistema inmune del recién nacido. (Contreras-Cuellar G, 2007) (Oliveira A, 2010).

En definitivo los resultados de este estudio muestra la presencia de enterobacterias BLEE en el hospital estudiado y la resistencia a las cefalosporinas. En ocasiones no 
permite muchas alternativas terapéuticas lo que puede llevar a una elevada morbimortalidad y por ende unl aumento de los costos hospitalarios por lo que estos resultados sirven para los tomadores de decisiones y los trabajadores de la salud construir programas educativos que promuevan la higiene especialmente el lavado de manos como un control de manera más efectiva de los comités de control de Infecciones asociadas a la atención en salud en los hospitales del país.

\section{BIBLIOGRAFÍA}

Alabi AS, Frielinhaus I, Kaba H, Küsters K, Huson MA, Kahl BC, et al.(2013) Retrospective analysis of antimicrobial resisitance and bacterial spectrum of infection in Gabon, Central Africa. BMC Infect Dis.13:455

Alanis AJ. (2005) Resistance to antibiotics: are we in the post-antibiotic era? Arch Med Res. Nov-Dec; 36(6):697-705.

Araya Fonseca C, Boza Cordero R, Arguedas Soto L, Badilla Baltodano G, Garcia Santamaria F. (2006) Infecciones nosocomiales por bacterias productoras de B lactamasas de espectro extendido: prevalencia, factores de riesgo y análisis molecular. AMC 49(2): 90-96

Contreras-Cuellar G, leal-Castro A, Prieto R, Carvajal-Hermida A. (2007) Device associated infections in a colombian neonatal intensive care unit. Rev Salud Publica 9 (3): 439-447

DeLisle S, Perl T. (2003) Vancomycin resistant enterococci: a road map on how to prevent the emergence and transmission of antimicrobial resistance. Chest 123: 504S-518S

Dielubanza EJ, Chaeffer AJ. (2011) Urinary Tract Infections in Women. Med Clin N Am. 95(1):27-41

Escalante-Montoya JC, Síme-Díaz A, Díaz-Vélez C. (2013) Características clínicas y epidemiológicas en pacientes con infección intrahospitalaria por bacterias productoras de betalactamasas de espectro extendido. Revista Peruana de Epidemiología Disponible en:http://www.redalyc.org/articulo.oa?id=2031285420 08

Giamarellou H. (2005) Multidrug resistance in Gram Negative bacteria that produce extended spectrum $\beta$ lactamasas (ESBLs). Clin Microbiol Infect; 11 (suppl. 4): $1-16$

Leal Aura L, Cortés JA, Ariasa G, Ovalleb MB, Saavedra SY, Buitragoa G, Escobar JA, Castro BE.(2013) Emergence of resistance to third generation cephalosporins by Enterobacteriaceae causing community-onset urinary tract infections in 
hospitals in Colombia. Enfermedades Infecciosas y microbiología clínica. (31) 5 , 298-303.

Mandomando I, Sigauque B, Morais I, Espasa M, Vallès X, Sacarlal J, et al. (2010) Antimicrobial drug resistance trends of bacteremia isolates in a rural hospital in Southern Mozambique. Am J Trop Med Hyg. 83:152-7

Muñoz Bellido JL. (2004) Betalactamasas de espectro ampliado ¿son un serio problema en España? Rev Esp Quimioterap 17:314-6

Murray PR, Baron EJ, Jorgensen JH, Pfaller MA, Yolken RH (2007). Manual of Clinical Microbiology. Ninth ed. ASM press. Washington, USA.

Oliveira A, Bettcher L. (2010) Epidemiological aspects of the ocurrence of vancomycin resistan enterococci. Rev Esc Enferm USP 44 (3): 716-721

Ortiz de Zárate MM, González del Castillo J, Jiménez AJ, Piñera Salmerón P, Llopis Roca F, Guardiola JM, Chanovas Borrás MR, Ruiz Grinspan M, García Lamberechts EJ, Esparza Cl, et al. (2003) Epidemiología de las infecciones atendidas en los servicios de urgencias hospitalarios y evolución durante la última década Emergencias: Revista de la Sociedad Española de Medicina de Urgencias y Emergencias. 25(5):368-378

Padgett D. Luque MT, Rivera DM, Galindo C, Zepeda LM, Hernandez AL. (2011). Resistencia Antimicrobiana en bacterias aisladas en el Instituto Hondureño de Seguridad Social. Rev Med Honduras. (79)3; 117-121Perez C. (2003). Antimicrobianos en unidades de cuidados intensivos: uso empirico. Rev Chil Infect. 20 (supl 1): S70-S73

Perianes-Díaza ME, Novo-Veleiroa I, Solís-Díaza K, Prolo-Acosta A, García-García I, Alonso-Claudio G. (2014) Bacteriemia por Escherichia coli y Klebsiella pneumoniae productoras de betalactamasas de espectro extendido: factores asociados a mortalidad y reingreso hospitalario. Medicina Clinica. (142)6:381-386

Rupp M, Fey P. (2003) Extended spectrum B lactamasa (ESBL) producing Enterobacteriaceae. Drugs; 63(4): 353-365

Schwaber MJ, Navon-Venezia S, Kaye KS, Ben-Ami R, Schwartz D, Carmeli Y. (2006) Clinical and economic impact of bacteremia with extended-spectrum- $\beta$-Lactamase-producing Enterobacteriacea. Antimicrob Agents Chemothe. 50:1257-1262

Seaman I. (2011) Costo social de las infecciones nosocomiales. Rev Med Hondureña (79) 3:155-160

Zemelman R, Valenzuela L, Dominguez M, Bello H, Gonzales G, Zemelman C. (2002) Detección de $B$ lactamasas de espectro extendido en el laboratorio de microbiología. Rev Chil Infect; 19 (supl 2): S 92-95 